\title{
Mn-, Ru-Coordinated Covalent Triazine Framework as Catalysts for Epoxidation of Cyclohexene
}

\author{
Xue Liu \\ Hubei Key Laboratory of Natural Products Research \\ and Development, College of Biological and \\ Pharmaceutical Sciences, China Three Gorges \\ University, Yichang 443002, China \\ e-mail:934349496@qq.com
}

\author{
Ling Zhang \\ Hubei Key Laboratory of Natural Products Research \\ and Development, College of Biological and \\ Pharmaceutical Sciences, China Three Gorges \\ University, Yichang 443002, China \\ e-mail:593305836@qq.com
}

\author{
Wei-Qiao Deng \\ State Key Laboratory of Molecular Reaction Dynamics, \\ Dalian National Laboratory for Clean Energy, Dalian \\ Institute of Chemical Physics Chinese Academy of \\ Science, Dalian 116023, China \\ e-mail: wqdeng@dicp.ac.cn
}

\author{
Nian-Yu Huang \\ Hubei Key Laboratory of Natural Products Research \\ and Development, College of Biological and \\ Pharmaceutical Sciences, China Three Gorges \\ University, Yichang 443002, China \\ e-mail: hny115@126.com (Corresponding author)
}

\begin{abstract}
The alkene epoxidation is an important reaction for organic synthesis and the catalytic epoxidation of cyclohexene can be widely used for the synthesis of fine chemicals. In this work, a new kind of heterogeneous catalysts, $\mathrm{Mn} / \mathrm{Ru}$ coorindated covalent triazine framework (Mn-CTF and Ru-CTF), for oxidation of cyclohexene to epoxy hexane were designed. The catalyst Mn-CTF/Ru-CTF was prepared via coordination of manganese chloride hydrate/ruthenium trichloride (III) hydrate with CTF, which was obtained by self-polymerization of 2 , 6pyridinedicarbonitrile. Reducing and oxidizing agents are isobutyraldehyde and molecular oxygen. The yield of epoxidation reaction of cyclohexene catalyzed by the MnCTF and Ru-CTF can be up to $97 \%$ and $99 \%$ under room temperature and atmospheric pressure, respectively.
\end{abstract}

Keywords-Covalent triazine framework; polymerization; cyclohexene epoxidation; catalysis; coordination

\section{INTRODUCTION}

Cyclohexane oxide is one of the important materials in organic synthesis. It can be used for the manufacture of ethylene glycol, detergents, emulsifiers, nonionic surfactants, antifreeze agents, plasticizers, lubricants and pesticides [1-2]. The epoxidation of cyclohexene has been studied extensively and many groups have made breakthrough over the past few decades [3-4]. However, in most cases, the catalyzed epoxidation of cyclohexene requires special conditions such as higher temperature or pressure, and large amounts of catalyst, etc. These conditions inevitably produced a lot of by-products , which not only resulted in a waste of raw materials, but also greatly enhanced the industrial production costs [5-8].

Covalent triazine frameworks (CTFs) have attracted widespread attention because of its high specific surface and low cost [9-10]. Subsequently, CTFs have been demonstrated potential applications in many fields, such as gas storage, organic dyes separation and heterogeneous catalysis. Therefore, it is no doubt that since a few years, more and more research groups became interested in the development of CTFs, and a lot of different types of covalent triazine frameworks have been synthesized [1112]. Especially, the excellent stability to acids, alkalis and high temperature of CTFs makes them one of the most studied catalyst supports. Recently, CTF-Pt was used as a catalyst by Ferdi Schuth's groups in methane oxidation to methanol and high catalytic efficiency was achieved [13]. Carine E. Chan-Thawa, etc. used one kind of CTFs as support for Pd nanoparticles, and the resulting Pd-CTF can be uesd as an efficient catalyst for glycerol oxidation [14].

In this work, we synthesized CTF by ionothermal trimerization of carbonitrile groups in molten $\mathrm{ZnCl}_{2}$ which acts as both solvent and catalyst at temperatures $400^{\circ} \mathrm{C} / 600^{\circ} \mathrm{C}$ and developed $\mathrm{Mn}$ (II) and $\mathrm{Ru}(\mathrm{II})$ coordinated CTF, and then used them as heterogeneous catalysts for the epoxidation of cyclohexene by molecular oxygen in the presence of isobutyraldehyde. The catalytic reaction can be achieved in ambient conditions. Moreover, facile operation of the reaction and high catalytic efficiency promise a potential application of the new CTFs as catalysts in cyclohexene epoxidation. 


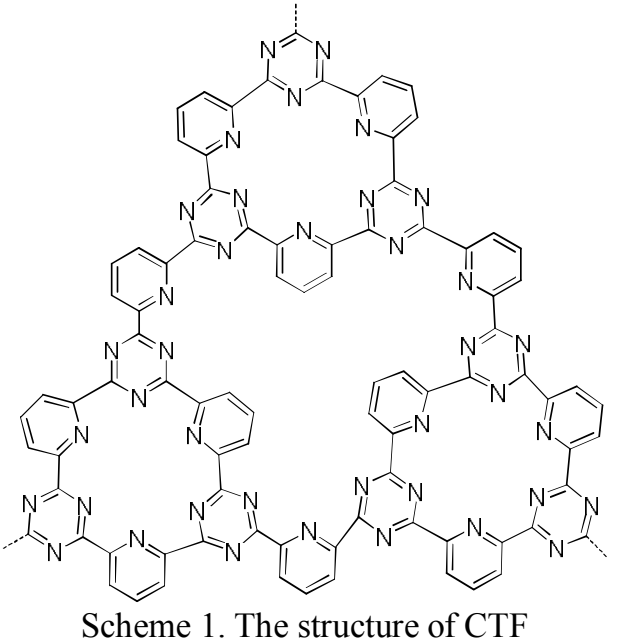

\section{EXPERIMENTAL}

A. Reagents and Physical Measurements

Unless otherwise noted, all reagents and materials were used without further purification. 2, 6Pyridinedicarbonitrile was purchased from Sigma-Aldrich Group Company. Anhydrous zinc chloride, ruthenium trichloride (III) hydrate $(38.0-42.0 \% \quad \mathrm{Ru}$ basis), manganese chloride hydrate and isobutyraldehyde (AR) were all purchased from Aladdin Reagent Company.

The synthesis of CTF was carried out in a muffle burner(TSX1200). The specific surface area of CTF was measured on adsorption instrument (Quantachrome QUADRASORB SI). The yield of cyclohexane oxide was tested on CG-MS spectrometry (QP2010SE).

\section{B. Synthesis Procedure of CTF}

Triazine-based material was synthesized as described elsewhere [15]. Specifically, 2,6-Pyridinedicarbonitrile (1g, $7.75 \mathrm{mmol})$ and anhydrous $\mathrm{ZnCl}_{2}(5.3 \mathrm{~g}, 39.0 \mathrm{mmol})$ were transferred into a pyrex ampoule $(3 \times 15 \mathrm{~cm})$ under an inert atmosphere. The ampule was evacuated by a vacuum pump, sealed, and heated to $400^{\circ} \mathrm{C}$ for $20 \mathrm{~h}$, after which the temperature was turnrd to $600^{\circ} \mathrm{C}$ for another $20 \mathrm{~h}$. The ampule was then cooled down to room temperature and opened carefully. Caution: the ampule is under pressure, which is released during-opening. The reaction mixture was subsequently grounded and then washed thoroughly with large amounts of water to remove most of the $\mathrm{ZnCl}_{2}$. Further stirring in diluted $\mathrm{HCl}$ for $15 \mathrm{~h}$ was carried out to remove the residual salt. After this purification step, the resulting black powder was filtered, washed successively with water and $\mathrm{THF}$, and dried in vacuum at $120^{\circ} \mathrm{C}$. Yield: $91 \%$.

\section{Synthesis Procedure of $\mathrm{MnCl}_{2}-\mathrm{CTF}\left(\mathrm{RuCl}_{3}-\mathrm{CTF}\right)$}

For $\mathrm{Mn}$ coordination, CTF $(500 \mathrm{mg})$ and $\mathrm{MnCl}_{2} \cdot 4 \mathrm{H}_{2} \mathrm{O}$ $(800 \mathrm{mg})$ were reacted in water under argon atmosphere for $6 \mathrm{~h}$ at $60^{\circ} \mathrm{C}$, then the reactant was cooled down to room temperature and filtered, washed with water and tetrahydrofuran, and dried overnight at $100^{\circ} \mathrm{C}$.

\section{Epoxidation of Cyclohexene Catalyzed by Mn-CTF (Ru-CTF)}

A solution of cyclohexene $(2 \mathrm{mmol})$ in dichloromethane $(5 \mathrm{~mL})$ was added to catalyst $(50 \mathrm{mg})$, isobutyraldehyde $(10 \mathrm{mmol})$. The reaction mixture was vigorous stirring at room temperature with oxygen in a balloon for $6 \mathrm{~h}, 12 \mathrm{~h}, 18 \mathrm{~h}$ and $24 \mathrm{~h}$. The reaction solution was filtered and the filtrate was diluted by dichloromethane and then tested on GC-MS. The residue was washed by dichloromethane and dried in a vacuum oven at $60^{\circ} \mathrm{C}$ for $12 \mathrm{~h}$ and recycling.

\section{RESULTS AND DISCUSSION}

\section{A. Nitrogen Adsorption and Desorption Isotherms}

Nitrogen adsorption and desorption isotherms of CTF, $\mathrm{Mn}-\mathrm{CTF}$, Ru-CTF were measured on an adsorption instrument before degassed at $200{ }^{\circ} \mathrm{C}$ for $10 \mathrm{~h}$. The analysis of porosity of CTF, Mn-CTF, Ru-CTF were listed in TABLE I. As shown in Fig .1, the nitrogen isotherms at $77 \mathrm{~K}$ indicate that the polymers are microporous. The surface area of the CTF, calculated by the Brunauer-Emmett-Teller (BET) equation, is $1680 \mathrm{~m}^{2} \mathrm{~g}$ 1. However, it reduced to 1466 and $1370 \mathrm{~m}^{2} \mathrm{~g}^{-1}$ after coordinated with $\mathrm{Mn}^{2+}$ and $\mathrm{Ru}^{3+}$. Moreover, the overall micropore volume are $0.69,0.44,0.48 \mathrm{~cm}^{3} \cdot \mathrm{g}^{-1}$ and the total pore volume are $0.86,0.74,0.77$, corresponding to $\mathrm{CTF}, \mathrm{Mn}-\mathrm{CTF}$ and Ru-CTF, respectively. The reduction of the porosity is ascribed to the clog of the micropores by metal ions.

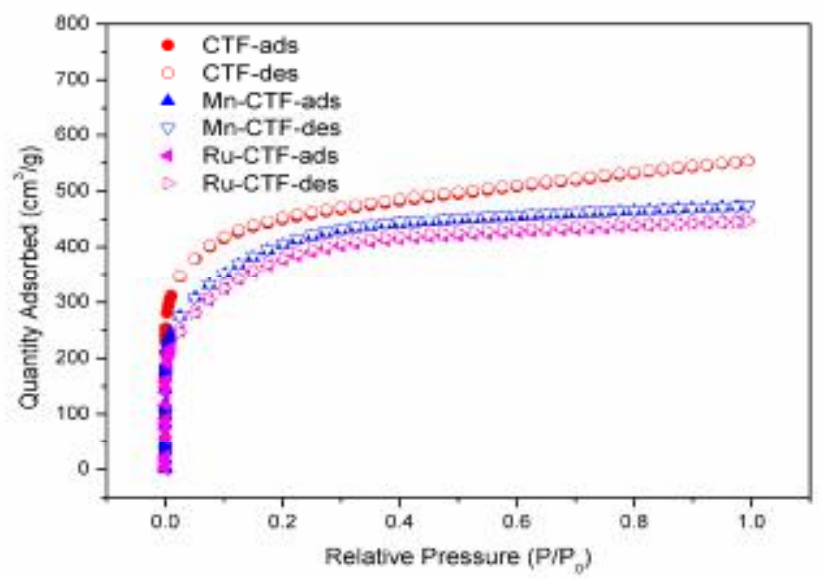

Figure 1. Argon adsorption (ads) and desorption (des) isotherms of CTF, Mn-CTF and Ru-CTF at 77K 


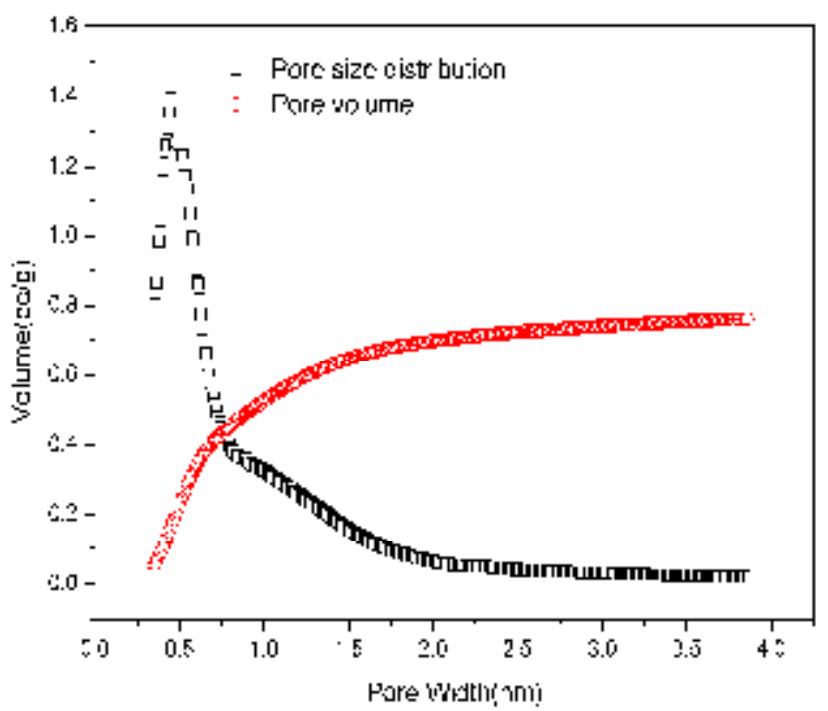

Figure 2. The pore size distribution and pore volume isotherms of CTF

TABLE I. CHARACTERIZATIONS OF CTFS BY NITROGEN SORPTION MEASUREMENTS

\begin{tabular}{cccc}
\hline Material & $\begin{array}{c}\mathrm{S}_{\mathrm{BET}} \\
\left(\mathrm{m}^{2} / \mathrm{g}\right)^{[\mathrm{a}]}\end{array}$ & $\begin{array}{c}\mathrm{V}_{\text {tot }} \\
\left(\mathrm{cm}^{3} / \mathrm{g}\right)^{[\mathrm{b}]}\end{array}$ & $\begin{array}{c}\mathrm{V}_{\text {micro }} \\
\left(\mathrm{cm}^{3} / \mathrm{g}\right)^{[\mathrm{c}]}\end{array}$ \\
\hline CTF & 1680 & 0.86 & 0.69 \\
Mn-CTF & 1466 & 0.74 & 0.44 \\
Ru-CTF & 1370 & 0.77 & 0.44
\end{tabular}

[a] Brunauer-Emmett-Teller (BET) surface area calculated over the pressure range $\left(\mathrm{P} / \mathrm{P}_{0}\right)$ 0.05-0.3.

[b] Total pore volume at $\mathrm{P} / \mathrm{P}_{0}=0.99$.

[c] Micropore volume calculated using the t-plot method.

\section{B. The Catalytic Performance of the Mn-CTF and Ru- CTF}

The catalytic performance of the Mn-CTF and RuCTF on cyclohexene epoxidation was listed in TABLE 2. Firstly, a benchmark experiment was carried out for comparison. The yield for epoxidation of cyclohexene was just $17 \%$ and $46 \%$ in the time of $6 \mathrm{~h}$ and $24 \mathrm{~h}$ without the use of catalyst (Entry 1 and 6). Only 35\% yield for cyclohexane oxide was achieved by Mn-CTF after $6 \mathrm{~h}$ at the room temperature and atmospheric pressure (Entry 2). However, the yields increased from $35 \%$ to $97 \%$ (Entry 25 ) when the reaction time prolonged from $6 \mathrm{~h}$ to $24 \mathrm{~h}$. Compared to Mn-CTF, Ru-CTF shows more excellent catalytic activity under the same conditions (6h) (Entry 2 and 7). Nevertheless, both high yields for cyclohexane oxide were obtained catalyzed by Mn-CTF $(97 \%)$ and RuCTF (99\%) after 24h (Entry 5 and 10). The high catalytic activity can be attributed to the enrichment of $\mathrm{O}_{2}$ concentrations near the catalytic center located in the pore structures of Mn/Ru-CTF [16].

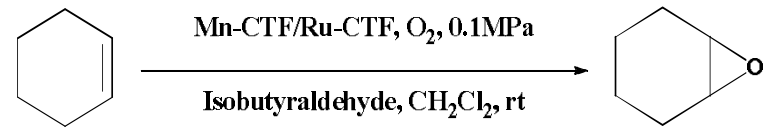

Scheme 2. Synthesis of the cyclohexane oxide

TABLE II. EPOXIDATION OF CYCLOHEXENE BY MOLECULAR OXYGEN IN THE PRESENCE OF Mn-CTF AND Ru-CTF WITH ISOBUTYRALDEHYDE

\begin{tabular}{|c|c|c|c|}
\hline Entry & $\begin{array}{c}\text { Time } \\
(\mathrm{h})\end{array}$ & $\begin{array}{c}\text { Catalyst } \\
(\mathrm{mg})\end{array}$ & $\begin{array}{c}\text { Yield } \\
(\%)\end{array}$ \\
\hline 1 & 6 & None & 17 \\
\hline 2 & 6 & Mn-CTF & 35 \\
\hline 3 & 12 & Mn-CTF & 64 \\
\hline 4 & 18 & Mn-CTF & 80 \\
\hline 5 & 24 & Mn-CTF & 97 \\
\hline 6 & 24 & None & 46 \\
\hline 7 & 6 & Ru-CTF & 55 \\
\hline 8 & 12 & Ru-CTF & 79 \\
\hline 9 & 18 & Ru-CTF & 89 \\
\hline 10 & 24 & Ru-CTF & 99 \\
\hline
\end{tabular}

\section{SUMMARY}

The special micropore structures of CTFs show good adsorption of gas molecules, which can enrich the concentrations of gaseous molecular near the catalytic center when they are used as catalysts. As a new kind of heterogeneous catalysts, Mn-CTF and Ru-CTF showed high catalytic efficiency for the cyclohexene oxidation. In addition, the excellent performance of the CTFs offers a useful way to find new catalysts with high efficiency and superior activity.

\section{ACKNOWLEDGMENT}

We gratefully acknowledge the financial support from the National Natural Science Foundation of China (No. 21373202).

\section{REFERENCES}

[1] M. Moosavifar, S. Tangestaninejad, M. Moghadam, V. Mirkhani, I. M. Baltork, "Host (nanocavity of zeolite Y)-guest (ruthenium(III) salophen complex) nanocomposite materials: An efficient and reusable catalyst for shape-selectiveepoxidation of linear alkenes with sodium periodate," Journal of Molecular Catalysis A: Chemical, vol. 377, 2013, pp. 92- 101. 
[2] Y. M. Cui, Y. Wang, Y. J. Cai, X. J. Long, W. Chen, "Syntheses and structures of N'-(5-bromo-2-hydroxybenzylidene)-4methoxybenzohydrazide and its dioxomolybdenum(VI) complex with catalytic epoxidation property," Journal of Coordination Chemistry, vol. 66, 2013, pp. 2325-2334.

[3] M. Zakeri, M. Moghadam, I. M. Baltork, S. Tangestaninejad, V. Mirkhani, A. R. Khosropour, M. Alizadeh, "Biomimetic epoxidation of alkenes with sodium periodate catalyzed by tetraphenylporphyrinatomanganese(III) chloride supported on multiwall carbon nanotubes,” Transition Met Chem, vol. 37, 2012, pp. $45-53$.

[4] A. C. Kalita, C. R. Marchal, R. Murugavel, "Cationic D4R zinc phosphate-anionic polyoxometalate hybrids: synthesis, spectra, structure and catalytic Studies," Dalton Trans, vol. 42, 2013, pp. 9755- 9763.

[5] L. L. Liu, H. X. Li, L. M. Wan, Z. G. Ren, H. F. Wang, J. P. Lang, "A Mn(III)-superoxo complex of a zwitterionic calix arene with an unprecedented linear end-on $\mathrm{Mn}(\mathrm{III})-\mathrm{O} 2$ arrangement and good catalytic performance for alkene epoxidation," Chem. Commun, vol. 47, 2011, pp. 11146- 11148 .

[6] D. G. Carlo, V. Lydia, S. Xavier, B. B. Jordi, A. Llobet, "New Dinuclear Ruthenium Complexes: Structure and Oxidative Catalysis," Inorg. Chem.,vol. 52, 2013, pp. 4335-4345.

[7] X. T. Zhou, H. B. Ji, "Biomimetic kinetics and mechanism of cyclohexene epoxidation catalyzed by metalloporphyrins," Chemical Engineering Journal, vol. 156, 2010, pp. 411- 417.

[8] A. Rezaeifard, R. Haddad, M. Jafarpour, M. Hakimi, "Catalytic Epoxidation Activity of Keplerate Polyoxomolybdate nanoball toward Aqueous Suspension of Olefins under Mild Aerobic Conditions," J. Am. Chem. Soc., vol. 135, 2013, pp. 10036- 10039.
[9] B. Asamanjoy, I. Boldog, C. Janiak, "Highly stable nanoporous covalent triazine-based frameworks with an adamantane core for carbon dioxide sorption and separation," J. Mater. Chem. A., vol. 1, 2013, pp. 14990- 14999.

[10] P. Kuhn, A. Thomas, M. Antonietti, "Toward Tailorable Porous Organic Polymer Networks: A High-Temperature Dynamic Polymerization Scheme Based on Aromatic Nitriles," Macromolecules, vol. 42, 2009, pp. 319-326.

[11] P. Katekomol, J. Roeser, M. Bojdys, J. Weber, A. Thomas, "Covalent Triazine Frameworks Prepared from 1,3,5 Tricyanobenzene," Chem. Mater, vol. 25, 2013, pp. 1542-1548.

[12] A. Modak, J. Mondal, M. Sasidharanb, A. Bhaumik, "Triazine functionalized ordered mesoporous polymer: a novel solid support for Pd-mediated $\mathrm{C}-\mathrm{C}$ cross-coupling reactions in water," Green Chem., vol. 13, 2011, pp. 1317-1331.

[13] R. Palkovits, M. Antonietti, P. Kuhn, A. Thomas, F. Schüth, "Solid Catalysts for the Selective Low-Temperature Oxidation of Methane to Methanol," Angew. Chem. Int. Ed., vol. 48, 2009, pp. 69096912.

[14] C. E. Thaw, A. Villa, P. Katekomol, D. Su, A. Thomas, L. Prati, "Covalent Triazine Framework as Catalytic Support for Liquid Phase Reaction,” Nano Lett., vol. 10, 2010, pp. 537-541.

[15] P. Kuhn, M. Antonietti, A. Thomas, Porous, "Covalent TriazineBased Fra meworks Prepared by Ionothermal Synthesis," Angew. Chem. Int. Ed., vol. 47, 2008, pp. 3450 -3453.

[16] Y. Xie, T. T. Wang, X. H. Liu, K. Zou, W. Q. Deng, "Capture and conversion of $\mathrm{CO} 2$ at ambient conditions by a conjugated microporous polymer," Nature Commun, vol. 4, 2013, pp. 19601966 